\title{
A Rellich-Kondrachov Theorem for Spiky Domains
}

\section{MARTy Ross}

\begin{abstract}
Suppose that $\Omega \subset \mathbb{R}^{n}$ is a bounded domain whose boundary can be locally represented as the graph of a (necessarily lower semicontinuous) function with $\Omega$ lying below its boundary. We show that if such boundary functions have everywhere defined limits, then, for $1 \leq p<\infty$, the Sobolev embedding $\iota: W^{1, p}(\Omega) \hookrightarrow L^{p}(\Omega)$ is compact. If $n=2$, then it is sufficient for the boundary functions to have left and right limits everywhere defined; in particular, for $n=2$ it is sufficient for $\partial \Omega$ to be locally connected or to have finite length. The same results hold for bounded domains locally diffeomorphic to such graphical domains, and thus in particular for starlike domains defined by radial functions with the appropriate properties.

For $n=3$ these results, in greater generality, follow easily from the literature. For $n=2$ the key property of the above domains to be established is that locally, after the application of interior diffeomorphisms, their boundaries can be written as the graphs of continuous functions; compactness then follows from standard theorems.
\end{abstract}

1. Survey. Suppose that $\Omega \subset \mathbb{R}^{n}$ is a bounded domain and, for $p \geq 1$, let $W^{1, p}(\Omega)$ be the Sobolev space of $L^{p}$ functions on $\Omega$ with $L^{p}$ weak derivatives, equipped with the norm

$$
\|u\|_{1, p} \equiv\|u\|_{L^{p}}+\|D u\|_{L^{p}} .
$$

Let $W_{0}^{1, p}(\Omega) \subset W^{1, p}(\Omega)$ be the completion of $C_{0}^{\infty}(\Omega)$ with respect to this norm. It is standard that the Rellich-Kondrachov Theorem holds for $W_{0}^{1, p}(\Omega)$, and in particular the embedding $W_{0}^{1, p}(\Omega) \hookrightarrow L^{p}(\Omega)$ is compact (see e.g. [Z, Section $2.5]$ ). It is of interest to know what conditions on (the boundary of) $\Omega$ ensure that, as well, 

the embedding $\iota: W^{1, p}(\Omega) \hookrightarrow L^{p}(\Omega)$ is compact?

Such a result with $p=2$, for instance, guarantees the discreteness of the Neumann spectrum for elliptic operators on $\Omega$ ([GT, Section 8.12], [CH1, Sections VI.2, 3]).

That (R-K) can fail to hold for quite reasonable domains is well known. This is illustrated by the "rooms and passages" set $D$ below ([CH2, Section VII.8.2], [F, Section 2.1]):

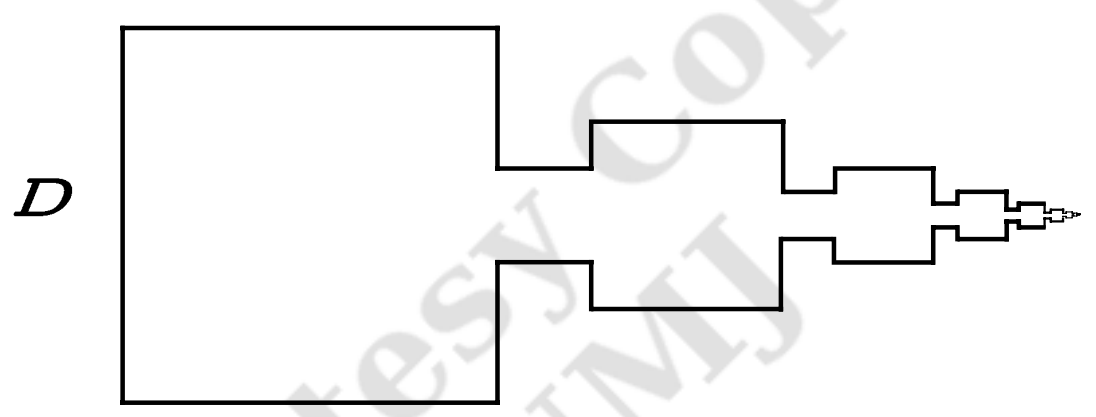

One can construct a sequence $\left\{u_{k}\right\} \subset W^{1, p}(D)$ with $u_{k}$ constant in the $k$ th room and dying out linearly in the adjoining passages, the constant adjusted so that $\left\|u_{k}\right\|_{L^{p}}=1$. If the passages are thin enough, then $\left\{\left\|D u_{k}\right\|_{L^{p}}\right\}$ will also be uniformly bounded, but clearly no subsequence of $\left\{u_{k}\right\}$ will converge in $L^{p}$. (For further examples of this type, see $[\mathrm{EH}]$ and the references cited in Section 1 of that paper).

The simplest general condition that ensures that (R-K) holds is for $\Omega$ to be Lipschitz: locally, $\partial \Omega$ can be written as the (Euclidean) graph of a Lipschitz function with $\Omega$ lying on one side of the graph. When such is the case, there is a bounded extension operator $E: W^{1, p}(\Omega) \rightarrow W_{0}^{1, p}(\widetilde{\Omega})$ for some $\widetilde{\Omega} \supset \Omega([\mathrm{EG}$, Section 4.4]), and the compactness of $\iota$ follows from the Rellich-Kondrachov Theorem for $W_{0}^{1, p}(\widetilde{\Omega})$. Notice that the boundary of $D$ above is a Jordan curve, has finite length (if desired), and fails to be Lipschitz in the neighbourhood of only one point.

As a weakening of the Lipschitz condition, one can consider those $\Omega$ satisfying a cone condition: for every $x \in \partial \Omega$ there is an open cone $C_{x} \subset \Omega$ with apex at $x$, all the cones being congruent (notice that a ball with deleted centre is not Lipschitz but does satisfy the cone condition). For cone domains there is an embedding 


$$
W^{1, p}(\Omega) \hookrightarrow L^{q}(\Omega) \quad \text { for some } q>p .
$$

By the same argument used for general $W_{0}^{1, p}(\Omega)$, this is sufficient to deduce the compactness of $\iota([\mathrm{Ad}$, Sections 5.4 and 6.2$])$.

An alternative generalization of Lipschitzness is the segment condition: for each $x \in \partial \Omega$ there is a neighbourhood $U$ of $x$ and a vector $v \in \mathbb{R}^{n}$ such that, if $y \in U \cap \bar{\Omega}$ and $0<t \leq 1$, then $y+t v \in \Omega$. Fraenkel notes that this is equivalent to being able to write $\Omega$ locally as lying on one side of the graph of a continuous function ([F, Theorem 3.3]). (Cusp domains and the deleted ball show that the cone condition and the segment condition are not comparable.)

(R-K) continues to hold for domains satisfying the segment/continuity condition, but this cannot be established by the previous argument: the example of an exponential cusp shows that (1) need not hold for domains satisfying the segment/continuity condition ([Ad, Theorem 5.32], [M1, pp. 204, 211]). Instead, the proof of (R-K) here is based upon showing, for each $\varepsilon>0$, the existence of an $\Omega^{\varepsilon} \Subset \Omega$ such that

$$
\|u\|_{L^{p}(\Omega)} \leq c\left(\varepsilon\|u\|_{W^{1, p}(\Omega)}+\|u\|_{L^{p}\left(\Omega^{\varepsilon}\right)}\right), \quad u \in W^{1, p}(\Omega),
$$

where $c=c(p, \Omega)([\mathrm{F}$, Section 5.3], [Ag, Theorem 3.2]); then one notes that (2) and the argument of $[\mathrm{Ag}$, Theorem 3.8] establishes the compactness of $\iota$ ([F, Theorem 5.3]). (Conversely, for $p=2$ at least, (R-K) implies (2): see [F, Remark 4, p. 414].) Also, Fraenkel points out that (2), and thus (R-K), continues to hold when $\Omega$ satisfies the segment/continuity condition after the application of local $C^{1}$ diffeomorphisms ([F, Section 5]): he refers to such domains as being of class $E$; this generalization establishes, for instance, that (R-K) will hold on domains with certain bent cusps.

Apart from the above, it appears difficult to determine geometric or topological conditions that guarantee (R-K). Maz'ja has established necessary and sufficient conditions in terms of capacity-like quantities ([M1, Section 4.8], [M2, Section 6]). However, except for rather concrete examples, these quantities appear to be difficult to interpret or compute.

In this paper we consider $\Omega \subset \mathbb{R}^{n}$ that can locally be written in terms of the graphs of possibly discontinuous functions: for each $a \in \partial \Omega$ there is a neighbourhood $U \ni a$ such that, in suitable orthogonal coordinates $(y, t)$ for $\mathbb{R}^{n-1} \times \mathbb{R}$, there is a function $h: B \rightarrow \mathbb{R}^{+}$on a ball $B \subset \mathbb{R}^{n-1}$ such that

$$
\Omega \cap U=\Omega_{h} \equiv \mathbb{R}^{n} \cap\{(y, t): y \in B, 0<t<h(y)\} .
$$




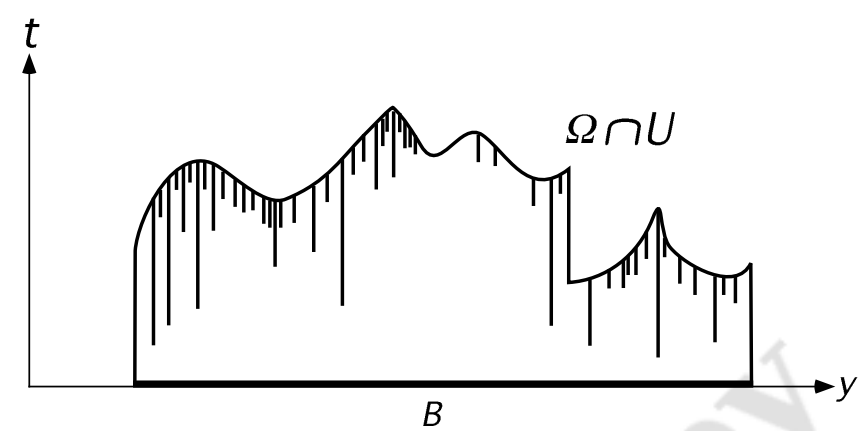

$\Omega$ being open is equivalent to such functions being lower semicontinuous, but we do not in general assume more (recall that the condition that all the functions be continuous is exactly the segment/continuity condition).

It is certainly not the case that (R-K) necessarily holds for such domains. As a simple and standard example, consider the domain $C \subset \mathbb{R}^{2}$ defined in terms of the one function $h:(-1,1) \rightarrow \mathbb{R}^{+}$where

$$
h(y)= \begin{cases}1 & \text { if } y=0 \text { or } y=\frac{1}{k}, k=1,2, \ldots \\ 2 & \text { otherwise }\end{cases}
$$

\section{As for $D$,
ergent subs
etrically $m$
Theorem.}

(a) Suppose $\Omega \subset \mathbb{R}^{n}$ is a bounded domain such that around each a $\in \partial \Omega$ there is a graphical representation of $\Omega$ of the form (3). Suppose that for each representative function $h: B \rightarrow \mathbb{R}^{+}$the limit

$$
\bar{h}(y) \equiv \lim _{z \rightarrow y} h(z)
$$

is everywhere defined. Then the embedding $\iota: W^{1, p}(\Omega) \hookrightarrow L^{p}(\Omega)$ is compact. 
(b) If $n=2$, then, for the conclusion of (a) to hold, it is sufficient that the one-sided limits

$$
\left\{\begin{array}{l}
h^{+}(y) \equiv \lim _{z \rightarrow y^{+}} h(z) \\
h^{-}(y) \equiv \lim _{z \rightarrow y^{-}} h(z)
\end{array}\right.
$$

exist everywhere.

\section{Remarks.}

(i) In (a), the function $\bar{h}(y)$ is continuous ([S, Ex6.16]), and thus we can think of $\Omega$ as being a domain defined by continuous functions to which countably many inward spikes have been added ([S, Ex21.33]). For $n>2$ these spikes are effectively removable (even for considerably worse $h$ ), making the proof of (a) in this case routine: see Section 2.

In (b), we are including, as well, the possibility of vertical segments.

(ii) A necessary and sufficient condition for (b) to be satisfied is that $\partial \Omega$ be locally connected. The necessity is easy to see. To see the sufficiency suppose that, say, the first limit in (5) is undefined at some $y$, and set

$$
\left\{\begin{array}{l}
h_{l}(y) \equiv \liminf _{z \rightarrow y^{+}} h(z), \\
h_{u}(y) \equiv \limsup _{z \rightarrow y^{+}} h(z) .
\end{array}\right.
$$

Then neighborhoods around $\left(y, h_{l}\right)$ of diameter less than $\min \left(h_{u}-h_{l}, h_{l}\right)$ will not be connected.

An alternative sufficient (though certainly not necessary) condition is that $\partial \Omega$ have finite length as a rectifiable curve (equivalently, in the sense of 1-dimensional Hausdorff measure). Similar to the previous argument, if at some point $y$ a limit in (5) doesn't exist, then the consequent zig-zag behaviour implies that $\partial \Omega$ will have infinite length around $\left(y, h_{l}\right)$.

(iii) The domain $D$ above shows that for $n=2$ in the non-graphical setting neither local connectivity nor finite length is in general sufficient for ( $\mathrm{R}$ $\mathrm{K})$ to hold. Nor are such conditions sufficient in the graphical setting for $n>2$. As an example to illustrate this, let $B \subset \mathbb{R}^{2}$ be a ball containing $D$ and let $A \subset \mathbb{R}^{3}$ be defined by the function $h: B \rightarrow \mathbb{R}^{+}$ where

$$
h(y)= \begin{cases}1 & y \in \partial D \\ 2 & \text { otherwise }\end{cases}
$$


Though $\partial A$ is locally connected and of finite area, one can easily show that (R-K) fails for $A$. Analogous domains of higher dimension can readily be constructed.

As indicated above, we will not have to work hard to prove (a) for dimensions greater than 2, but it will be seen that the proof given fundamentally fails in dimension 2: a completely different argument is needed for (b), even if one assumes the representatives have two-sided limits. To this end, we consider a natural generalization of Fraenkel's class $E$ :

Definition. Suppose that $\Omega \subset \mathbb{R}^{n}$ is open.

(i) A function $F: \Omega \rightarrow \mathbb{R}^{n}$ is ray-Lipschitz if there is a $K>0$ such that

$$
|F(x)-F(y)| \leq K|x-y| \quad x, y \in \Omega
$$

whenever the ray connecting $x$ to $y$ is contained in $\Omega$. We write $\operatorname{Lip}_{\mathrm{r}} F$ for the smallest $K$ for which (6) holds. If $F$ is injective and $F^{-1}$ is also ray-Lipschitz, then $F$ is ray-biLipschitz.

(ii) We say $\Omega$ is of class $\mathcal{F}$ if, for each $a \in \partial \Omega$, there is a neighbourhood $U \ni a$ and a ray-biLipschitz function $F: \Omega \cap U \rightarrow V$ onto a domain $V \subset \mathbb{R}^{n}$ satisfying the segment/continuity condition.

Clearly a ray-Lipschitz function on a convex domain is automatically Lipschitz. In general, $F$ is ray-Lipschitz iff $F$ is locally Lipschitz with a global bound on the local Lipschitz constant, and then

$$
\operatorname{Lip}_{\mathrm{r}} F=\max |D F|
$$

In particular, the (almost everywhere defined) Jacobian of a ray-Lipschitz map $F$ can be globally bounded in terms of $\operatorname{Lip}_{\mathrm{r}} F$, and thus if $F$ is ray-biLipschitz, then any inequality of the form (2) is preserved by $F$ (see [Z, Section 2.2]). Consequently, by the compactness of $\partial \Omega$,

\section{(R-K) will hold on any domain of class $\mathcal{F}$.}

There are two aspects in which the class $\mathcal{F}$ generalizes $E$. Firstly (and not critically for us), we are allowing Lipschitz rather than just $C^{1}$ deformations ([F, p. 414] notes this possibility). More importantly, the deformations do not have to extend nicely, even continuously, to pieces of $\partial \Omega$. This permits the following result, the key to the proof of Part (b) of the Theorem: 
Lemma. Suppose that $\Omega \subset \mathbb{R}^{2}$ is a bounded domain with local representations of the form (3) for which the limits in (5) are everywhere defined. Then $\Omega$ is of class $\mathcal{F}$.

\section{Remarks.}

(iv) By consideration of smooth domains to which a single inward spike has been added, it can be seen that this Lemma is fundamentally false in higher dimensions.

(v) As for the class $E$, we note that the class $\mathcal{F}$ and inequalities of the form (2) are preserved by ray-Lipschitz diffeomorphisms. Consequently, (R-K) will hold for any $\Omega \subset \mathbb{R}^{n}$ that, after the application of such diffeomorphisms, satisfies the hypotheses of the Theorem. In particular, it is natural to consider bounded starlike domains. Such a domain $\Omega$ can be written in spherical coordinates $(\rho, \omega)$ in terms of a radial function $h: \mathbb{S}^{n-1} \rightarrow \mathbb{R}^{+}$:

$$
\Omega=\left\{(\rho, \omega): \omega \in \mathbb{S}^{n-1}, \quad 0 \leq \rho<h(\omega)\right\} .
$$

If the limits of $h$ exist in the appropriate sense, then (R-K) holds for $\Omega$.

\section{Proofs.}

Proof of Part (a) of the Theorem, $n>2$. It is actually enough to hypothesize, for each representative $h$, the existence of a continuous $\bar{h}$ such that $\bar{h}=h$ except on a set of Hausdorff $\mathcal{H}^{n-2}$ measure zero: by Remark (i) above, this covers the case of the Theorem for $n>2$.

We can use $\bar{h}$ to define a domain $\widetilde{\Omega} \supset \Omega$ satisfying the segment/continuity condition and for which $\mathcal{H}^{n-1}(\widetilde{\Omega}-\Omega)=0$. Now, a standard characterization of a function $u \in W^{1, p}(\Omega)$ is that $u$ be absolutely continuous on (Lebesgue) almost all connected line segments parallel to the coordinate axes, with $L^{p}$ classical partial derivatives $\left(\left[\mathrm{Z}\right.\right.$, Theorem 2.1.4]). As a consequence $W^{1, p}(\widetilde{\Omega})=W^{1, p}(\Omega)$, and thus (R-K) holds for $\Omega$.

Notice that this argument is vacuous for $n=2$. In fact, the domain $C$ above shows that no such simple argument can work in dimension 2 .

Proof of the Lemma and Part (b) of the Theorem. As indicated in Section 1, (b) follows immediately from the Lemma, and of course the $n=2$ case of (a) follows from (b).

In setting up the proof of the Lemma, we can assume that $\Omega=\Omega_{h}$ is a single graphical domain of the form (3). If $h:[-R, R] \rightarrow \mathbb{R}^{+}$is continuous, then there 
is nothing to prove. In general, we shall show that there is a ray-biLipschitz diffeomorphism $F: \Omega_{h} \rightarrow \Omega_{h^{*}}$ with $h^{*}$ continuous. (Actually, when $\Omega$ is the union of more than one graphical domain it is not hard to construct a global diffeomorphism. Since $\partial \Omega$ is compact, we need only consider a finite number of graphical domains; from the form of the local diffeomorphisms below, it is clear that we can compose them to give the desired global map).

SteP 1. As a first case, suppose that $h$ is continuous everywhere except at $y=0$ and that $h^{-}(0)=h(0)$. By lower semicontinuity,

$$
h^{+}(0)>h(0) .
$$

Consequently, if $S<R$ is small enough, then

$$
0<y \leq S \Longrightarrow h(y)>h(0)
$$

Next, choose a $C^{1}$ function $\ell:\left[h(0), h^{+}(0)\right] \rightarrow[0, S]$ satisfying

$$
\left\{\begin{array}{l}
\ell(h(0))=S, \\
\ell\left(h^{+}(0)\right)=0, \\
\ell \text { is strictly decreasing, } \\
\ell^{-1}(y)<h(y) \text { whenever } 0<y \leq S .
\end{array}\right.
$$

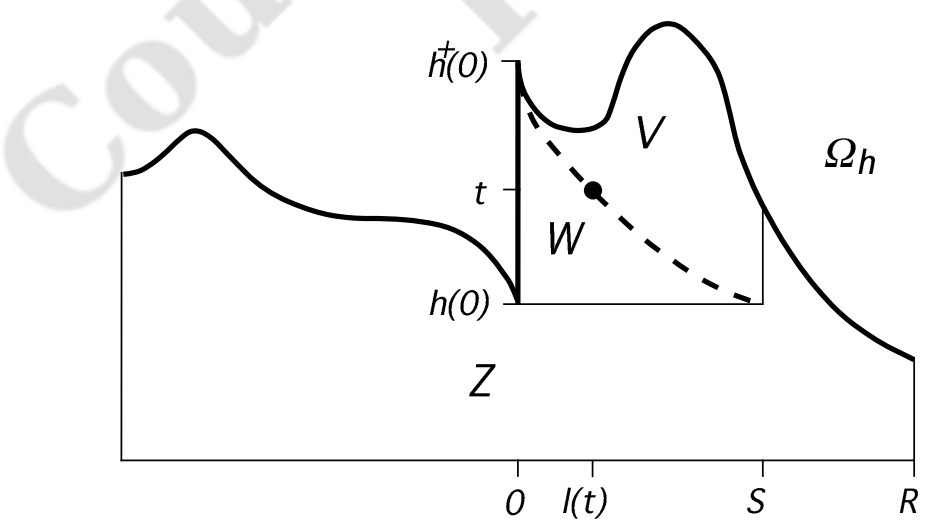

Next, fix $\eta$ with $0<\eta<S$ and define $m:\left[h(0), h^{+}(0)\right] \rightarrow[0, S]$ by

$$
m(t)=\eta\left(1-\frac{\ell}{S}\right)
$$


We now decompose $\Omega_{h}$ as follows:

$$
\left\{\begin{array}{l}
V=\Omega_{h} \cap\left\{(y, t): 0<y<S \text { and } \ell^{-1}(y)<t\right\} \\
W=\Omega_{h} \cap\left\{(y, t): 0<y<S \text { and } h(0)<t \leq \ell^{-1}(y)\right\} \\
Z=\Omega_{h}-(V \cup W)
\end{array}\right.
$$

Finally, we can define the desired mapping $F: \Omega_{h} \rightarrow \mathbb{R}^{2}$ to be the identity on $Z$ and in general

$$
F(x)=F(y, t)=(f(x), t)
$$

where

$$
f(x)=f(y, t)= \begin{cases}y & x \in Z, \\ \left(1-\frac{\eta}{S}\right) y+\eta & x \in V, \\ y+m(t) & x \in W .\end{cases}
$$

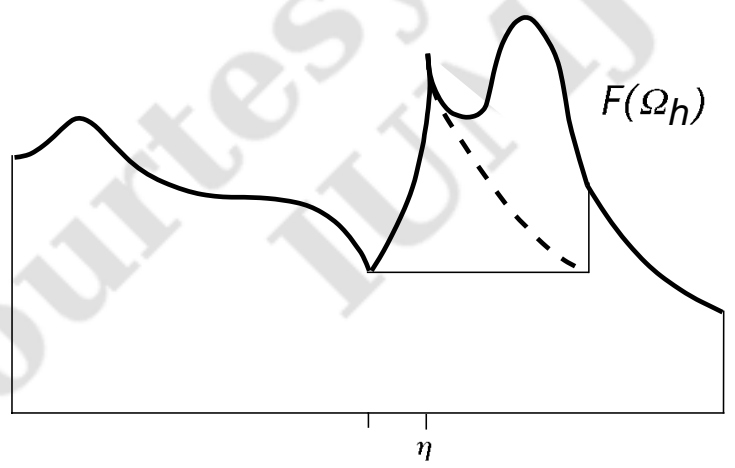

Geometrically, $F$ is a linear contraction/translation along horizontal lines; in particular, $F_{\left.\right|_{V}}$ is contraction by a factor $1-\eta / S$ towards the line $y=S$, and thus the images of vertical lines in $V$ are vertical. From this it is not hard to see that $F$ is a homeomorphism of $\Omega_{h}$ onto a graphical domain $\Omega_{h^{*}}$, where

$$
h^{*}(y)=\left\{\begin{array}{lc}
h(y) & -R \leq y \leq 0, \\
m^{-1}(y) & 0<y \leq \eta, \\
h\left(\frac{S(y-\eta)}{S-\eta}\right) & \eta<y \leq S, \\
h(y) & S<y \leq R .
\end{array}\right.
$$


Notice that, as desired, $h^{*}$ is continuous.

To show that $F$ is ray-biLipschitz reudces to showing that $F_{\mid W}$ is raybiLipschitz. But

$$
D F_{\mid W}=\left(\begin{array}{cc}
1 & -\eta \ell^{\prime} \\
0 & 1
\end{array}\right) .
$$

Thus, $D F$ is ray-biLipschitz by (7), with

$$
\left\{\begin{array}{l}
\operatorname{Lip}_{r} F \leq 1+c \eta \\
\operatorname{Lip}_{\mathrm{r}} F^{-1} \leq 1+c \eta
\end{array} \quad \eta<\frac{S}{2}, S=S\left(\Omega_{h}\right), c=c\left(\Omega_{h}\right) .\right.
$$

(One can take $c=(1 / S) \max \left(2, \max \left|\ell^{\prime}\right|\right)$.)

STEP 2. We now consider the general case, constructing the desired map $F$. The idea is to inductively apply the argument of Step 1 , transforming the spikes/segments into cusps one by one. The concern is to do this carefully enough to ensure that no new discontinuities are created in the limit.

Let

$$
A_{1}=[-R, R] \cap\left\{y: h^{+}(y)-h(y) \geq 1 \text { and/or } \quad h^{-}(y)-h(y) \geq 1\right\} .
$$

Since $h^{+}$and $h^{-}$are everywhere defined, $A_{1}$ is finite. Of $A_{1}=\varnothing$, then we set $h_{1}=h$. Otherwise, we sequentially apply the argument of Step 1 a finite number of times to give a ray-biLipschitz $G_{1}: \Omega_{h} \rightarrow \Omega_{h_{1}}$, where $h_{1}$ has no discontinuities of size 1 or greater, and where $h_{1}^{+}$and $h_{1}^{-}$are everywhere defined. Consequently, there is a $\delta_{1}>0$ such that

$$
|z-y|<2 \delta_{1} \Rightarrow\left|h_{1}(z)-h_{1}(y)\right|<1 .
$$

Furthermore, by (8) we can make choices of $\eta$ small enough to ensure that

$$
\operatorname{Lip}_{\mathrm{r}} G_{1}, \operatorname{Lip}_{\mathrm{r}} G_{1}^{-1} \leq 2 .
$$

Next, let

$$
A_{2}=[-R, R] \cap\left\{y: h_{1}^{+}(y)-h(y) \geq \frac{1}{2} \quad \text { and } / \text { or } \quad h_{1}^{-}(y)-h(y) \geq \frac{1}{2}\right\} .
$$

As above, we can obtain $G_{2}: \Omega_{h_{1}} \rightarrow \Omega_{h_{2}}$ and $\delta_{2}>0$ with

$$
\operatorname{Lip}_{\mathrm{r}} G_{2}, \operatorname{Lip}_{\mathrm{r}} G_{2}^{-1} \leq \frac{5}{4}
$$

and

$$
|z-y|<\frac{3}{2} \delta_{2} \Rightarrow\left|h_{2}(z)-h_{2}(y)\right|<\frac{1}{2} .
$$

We can also ensure that (9) remains true with a slightly smaller $\delta$ : $|z-y|<\frac{3}{2} \delta_{1} \Rightarrow\left|h_{2}(z)-h_{2}(y)\right|<1$. (We just have to ensure that $h_{1}$ is not 
shifted too much horizontally in the construction of $h_{2}$; if $R_{1}, \ldots, R_{N}$ are the radii of the neighbourhoods of the points in $A_{2}$, then it is sufficient to take $\left.\sum R_{j}<\delta_{1} / 4\right)$.

Proceeding inductively, we obtain $G_{k}: \Omega_{h_{k-1}} \rightarrow \Omega_{h_{k}}$ and $\delta_{k}>0$ such that

$$
\operatorname{Lip}_{\mathrm{r}} G_{k}, \operatorname{Lip}_{\mathrm{r}} G_{k}^{-1} \leq 1+\frac{1}{k^{2}}
$$

and

$$
|z-y|<\left(1+\frac{1}{k}\right) \delta_{j} \Rightarrow\left|h_{k}(z)-h_{k}(y)\right|<\frac{1}{j}, \quad j=1,2, \ldots, k .
$$

Define $F_{k}: \Omega_{h} \rightarrow \Omega_{h_{k}}$ by

$$
F_{k}=G_{k} \circ G_{k-1} \circ \cdots G_{1} .
$$

By the construction in Step 1, each $G_{k}$ can be defined to be the identity except near the graph of $h$ (i.e., near the top piece of $\partial \Omega_{h_{k}}$ ). Consequently, we can apply the procedure above so that each $F_{k}$ is eventually the identity: for any $\tilde{\Omega}$ with $\operatorname{dist}(\tilde{\Omega}, \operatorname{graph} h)>0$ there is a $K$ such that

$$
k \geq K \Rightarrow G_{k} \text { is the identity on } F_{k-1}(\tilde{\Omega}), \quad K=K(\tilde{\Omega}) .
$$

We can therefore define $F: \Omega_{h} \rightarrow \mathbb{R}^{2}$ by

$$
F(x) \equiv \lim _{k \rightarrow \infty} F_{k}(x), \quad x \in \Omega_{h} .
$$

STEP 3. We show that $F$ given by (13) is a ray-biLipschitz diffeomorphism onto a graphical domain $\Omega_{h^{*}}$ with $h^{*}$ continuous. To begin, it easily follows from (12) that $F$ is a homeomorphism onto a domain $\Omega^{*}=F\left(\Omega_{h}\right)$. Next, by (10) together with the chain rule for Lipschitz functions ([Z, Theorem 2.2.2]), we have the estimates

$$
\operatorname{Lip}_{\mathrm{r}} F_{k}, \operatorname{Lip}_{\mathrm{r}} F_{k}^{-1} \leq \prod_{j=1}^{k}\left(1+\frac{1}{j^{2}}\right)<\prod_{j=1}^{\infty}\left(1+\frac{1}{j^{2}}\right) \equiv L<\infty .
$$

So, by (13), $F$ is ray-biLipschitz.

It remains to show $\Omega^{*}=\Omega_{h^{*}}$ for some continuous $h^{*}$. Suppose that $x=$ $(y, t) \in \Omega^{*}$. We shall show that the sets

$$
R_{j} \equiv \mathbb{R}^{2} \cap\left\{(z, s):|z-y|<\frac{\delta_{j}}{2} \text { and } 0<s \leq t-\frac{2}{j}\right\}, \quad j=1,2, \ldots
$$

satisfy

$$
R_{j} \subset \Omega^{*}
$$


That $\Omega^{*}=\Omega_{h^{*}}$ is graphical is then immediate, and it also easily follows that $h^{*}$ is continuous: $h^{*}$ is automatically lower semicontinous, and (15) implies that $h^{*}$ is upper semicontinuous.

To prove (15), set $p=F^{-1}(x)$ and note that (12) implies $F_{k}(p)=x$ for all large $k$. Now, fixing $j$, we have $R_{j} \subset \Omega_{h_{k}}$. In fact, setting

$$
\varepsilon=\min \left(\frac{1}{2 j}, \frac{\delta_{j}}{2}\right) \text {, }
$$

(11) implies

$$
R_{j} \subset \Omega_{h_{k}} \cap\left\{x: \operatorname{dist}\left(x, \text { graph } h_{k}\right)>\varepsilon\right\}, \quad k \text { large. }
$$

Then, setting

$$
\tilde{\Omega}=\Omega_{h} \cap\left\{p: \operatorname{dist}(p, \operatorname{graph} h)>\frac{\varepsilon}{L}\right\},
$$

(14) implies that $R_{j} \subset F_{k}(\tilde{\Omega})$ for large $k$. So, by (12) and (13), $R_{j} \subset F\left(\Omega_{h}\right)=\Omega^{*}$, establishing (15) and completing the proof.

Acknowledgments. I would very much like to thank the referee for his detailed and helpful suggestions.

\section{REFERENCES}

[Ad] R. A. Adams, Sobolev Spaces, Academic Press, Orlando, Florida, 1975.

[Ag] S. AGmon, Lectures on Elliptic Boundary Value Prolems, van Nostrand, Princeton, 1965.

[CH1] R. Courant And D. Hilbert, Methods of Matehmatical Physics I, Interscience, New York, 1953.

[CH2] R. Courant And D. Hilbert, Methoden der Mathematischen Physik II, Zweit Auf., Springer-Verlag, Berlin, 1968.

[EG] L. C. Evans And R. F. GARIEPy, Measure Theory and Foine Properties of Functions, CRC Press, Boca Raton, Florida, 1992.

[EH] W. D. Evans AND D. J. HARRIS, Fractals, trees and the Neumann Laplacian, Math. Ann. 296 (1993), 493-527.

[F] L. E. FRAENKEL, On regularity of the boundary in the theory of Sobolev spaces, Proc. London Math. Soc. 39 (1979), 385-427.

[GT] D. Gilbarg And N. S. Trudinger, Elliptic Partial Differential Equations of Second Order, 2nd. edition, Springer-Verlag, New York, 1983.

[M1] V. G. MAZ'JA, Sobolev Spaces, Springer-Verlag, New York, 1980.

[M2] V. G. MAZ'JA, On Neumann's problem in domains with nonregular boundary, Siberian Math. J. 9 (1968), 990-1012.

[S] M. SPIVAK, Calculus, 2nd edition, Publish or Perish, Houston, TX, 1980. 
[Z] W. P. ZIEMER, Weakly Differentiable Functions, Springer-Verlag, New York, 1980.

Institute of Antarctic and Southern Ocean Studies

University of Tasmania

Box 252-77

Hobart, 7001, AUSTRALIA

EMAIL: marty.ross@utas.edu.au

Received: July 1st, 1997; revised: April 27th, 1998. 